\title{
Hybrid Conditional Planning using Answer Set Programming
}

\author{
Ibrahim Faruk Yalciner, Ahmed Nouman, Volkan Patoglu, and Esra Erdem \\ Faculty of Engineering and Natural Sciences, Sabanci University, Istanbul, Turkey \\ (e-mail: fyalciner, ahmednouman, vpatoglu, esraerdem@sabanciuniv.edu) \\ submitted 1 January 2003; revised 1 January 2003; accepted 1 January 2003
}

\begin{abstract}
We introduce a parallel offline algorithm for computing hybrid conditional plans, called HCP-ASP, oriented towards robotics applications. HCP-ASP relies on modeling actuation actions and sensing actions in an expressive nonmonotonic language of answer set programming (ASP), and computation of the branches of a conditional plan in parallel using an ASP solver. In particular, thanks to external atoms, continuous feasibility checks (like collision checks) are embedded into formal representations of actuation actions and sensing actions in ASP; and thus each branch of a hybrid conditional plan describes a feasible execution of actions to reach their goals. Utilizing nonmonotonic constructs and nondeterministic choices, partial knowledge about states and nondeterministic effects of sensing actions can be explicitly formalized in ASP; and thus each branch of a conditional plan can be computed by an ASP solver without necessitating a conformant planner and an ordering of sensing actions in advance. We apply our method in a service robotics domain and report experimental evaluations. Furthermore, we present performance comparisons with other compilation based conditional planners on standardized benchmark domains. This paper is under consideration for acceptance in TPLP.
\end{abstract}

KEYWORDS: Conditional planning, hybrid planning, answer set programming, cognitive robotics

\section{Introduction}

Conditional planning is concerned with reaching goals from an initial state, in the presence of incomplete knowledge and sensing actions (Warren 1976; Peot and Smith 1992; Pryor and Collins 1996). Since all contingencies are considered in advance, a conditional plan is essentially a tree of actions where the root represents the initial state, leaves represent goal states, and each branch of the tree from the root to a leaf represents a possible execution of actuation actions and sensing actions to reach a goal state. The existence of a conditional plan is an intractable problem: for polynomially bounded plans with partial observability, it is PSPACE-complete (Baral et al. 1999). Recall that classical planning considers complete knowledge and full observability, and it is NP-complete for polynomially bounded plans (Erol et al. 1995).

We are concerned about computing conditional plans that are executable by robots. In real-life robotic applications, applicability of high-level planning techniques (like navigation of a robot) necessitate low-level feasibility checks (like collision checks). That a valid plan exists does not mean that the plan is executable by a robot. Therefore, some sort of hybrid planning is needed that combines planning with feasibility checks. This is a challenging problem, considering that high-level planning is done over discrete representations of the world whereas feasibility checks are performed over continuous spaces of robotic configurations.

Along these lines, several hybrid planning approaches have been proposed in the literature, where classical planning is integrated with low-level feasibility checks: by modifying the search 
algorithms of the planners by embedding feasibility checks to compute successor states, or by modifying the representations of the domain descriptions by embedding feasibility checks into preconditions (and other constraints) via semantic attachments.

In this paper, we consider both sorts of challenges, and introduce a novel method for hybrid robotic planning under incomplete knowledge that relies on the following contributions.

- In conditional planning, in addition to familiar actuation actions, sensing actions are also considered as part of the planning problem, and different plans are computed according to all possible outcomes of such sensing actions. As a result, a conditional plan is a tree of actions (Figure 1), where the branching occurs at vertices that characterize sensing actions, while other vertices characterize actuation actions.



Fig. 1: A sample conditional plan

We introduce an offline compilation-based method to incrementally construct a conditional plan, that utilizes two sorts of parallelization.

(i) Parts of branches (i.e., sequences of actuation and sensing actions that reach a goal state from a state where a sensing action occurs) are computed in parallel using classical planners and combined into subtrees as they are computed.

(ii) Subtrees of a conditional plan are computed in parallel according to (i) and then combined into larger subtrees. During this incremental construction, previously computed subtrees are re-used to improve computational efficiency.

- Since the existing classical planning approaches consider full observability and actuation actions only, they cannot be used in computation of branches in (i) which also involve partial observability and sensing actions. To handle this challenge, we introduce a novel method to represent partial states (defined over functional fluents) and sensing actions using answer set programming (ASP) so that hybrid sequential plans of actuation actions and sensing actions can be computed using the existing ASP solvers.

- Cardinality expressions are used to represent "unknowns" (e.g., when the value of a functional fluent is not known) in a partially observed state.

- Defaults are used to further describe assumptions about partially observed worlds (e.g., unless the location of an object is known, the object is assumed to be not on the table since the table is fully observable).

- Defaults are used to describe exogenous occurrences (and non-occurrences) of sensing actions. In this way, there is no need to decide on the order of sensing actions in advance (before conditional planning).

- Nondeterministic outcomes of sensing actions are represented using nondeterministic choices and cardinality constraints. 
Since our method plans also for the sensing actions required to reach the goal in every branch of a conditional plan, unlike the existing compilation-based conditional planning methods, there is no need to decide on the order of sensing actions in advance (before conditional planning) or separate their computation from that of the actuation actions. Also, since our method allows for modeling sensing actions as nondeterministic actions, unlike the existing compilation-based conditional planning methods, there is no need to use conformant planners to compute parts of branches that consist of actuation actions only and that occur between two sensing actions.

- To incrementally compute hybrid conditional plans for robotic applications as described above, we adopt a representation-based approach to embed low-level feasibility checks into action descriptions so that the computed sequences of actions in (i), and thus conditional plans, are more likely to be physically implementable. In particular, we extend our representation-based approach to sensing actions as well. Note that, since the existing representation-based approaches consider deterministic actuation actions only, they cannot be adopted for the computation of branches in (i) which also involve sensing actions.

We evaluate our method experimentally over a real-world service robotics domain where a bimanual mobile manipulator sets up a kitchen table in the presence of incomplete knowledge about kitchenware. Furthermore, we present performance comparisons with other compilation based conditional planners on standardized benchmark domains.

\section{Related Work}

There is a variety of conditional planners, despite the hardness of computing conditional plans: even for polynomially bounded plans with limited number of sensing actions, its complexity is PSPACE-complete (Baral et al. 1999). Some of these planners are online, such as CLG (Albore et al. 2009), K-Planner (Bonet and Geffner 2011), SDR (Brafman and Shani 2012), HCP (Maliah et al. 2014) and CPOR (Komarnitsky and Shani 2016). Since sensing is done online, these solvers do not end up handling many contingencies and thus sometimes do not reach a goal state. Some of the conditional planners are offline, such as Contingent-FF (Hoffmann and Brafman 2005), POND (Bryce et al. 2006), PKS (Petrick and Bacchus 2002), ASCP (Tu et al. 2007), DNFct (To et al. 2011), PO-PRP (Muise et al. 2014), HCplan (Nouman et al. 2016). These planners construct whole conditional plans with decision points for sensing outcomes, where each execution via a branch of the plan leads to a goal state. Our planner is an offline planner.

Offline conditional planners can be further classified into two groups: search-based approaches and compilation-based approaches. The former group views conditional planning as a nondeterministic search problem in belief space (e.g., Contingent-FF, PKS, POND), whereas most of the planners in the latter group compiles a conditional planning problem into many conformant planning problems by deciding for the order of sensing actions and then computing the branches of the conditional plan by means of conformant planning (e.g., CLG, DNFct, PO-PRP). Recall that conformant planning considers incomplete initial state and no observability, and its aim is to find an action sequence that reaches the goal for every possible initial state (Goldman and Boddy 1996). Our planner can be characterized as a compilation-based planner since we compile a conditional planning problem into many sequential planning problems.

Essentially, we propose a generic hybrid conditional planning algorithm, called HCP-ASP, with an offline compilation-based approach. Except for HCplan, it is different from other offline 
Table 1: A comparison of offline compilation-based conditional planners

\begin{tabular}{|c||r|r|r|r|}
\hline Name & Hybrid & Parallel & Mode & Language (variant) \\
\hline CLG & no & no & offline/online & PDDL \\
\hline PO-PRP & no & no & offline & PDDL/STRIPS \\
\hline DNFct & no & no & offline & PDDL \\
\hline HCplan & yes & yes & offline & $\mathscr{C}+$ \\
\hline HCP-ASP & yes & yes & offline & ASP \\
\hline
\end{tabular}

compilation-based approaches, since it is hybrid (so low-level feasibility checks are integrated into conditional planning), it models multi-valued sensing actions as nondeterministic actions (so ordering of sensing actions and solving conformant planning problems are not needed as in the related work), and it allows for concurrency of actuation actions. See Table 1 for a comparison.

HCP-ASP has similarities with HCplan: they both compute hybrid conditional plans, utilize parallel computation of branches, use nonmonotonic default constructs to represent nonoccurrences of sensing actions, and outcomes of sensing actions are determined nondeterministically. They do not have to decide for the order of sensing actions in advance. On the other hand, the input language for HCplan is $\mathscr{C}+$ (Giunchiglia et al. 2004), while it is answer set programming (ASP) (Brewka et al. 2016) for HCP-ASP. In HCplan, outcomes of sensing actions are nondeterministically decided by external computations, whereas in HCP-ASP they are nondeterministically decided by ASP. Moreover, HCP-ASP keeps track of visited belief states and avoids recomputing subtrees of a hybrid conditional plan.

Although not compilation-based, the offline conditional planner ASCP is also related to HCPASP: It is not hybrid, not parallel, and its input language is the action language $\mathscr{A}_{K}^{c}$; but it uses ASP to compute conditional plans. Based on 0-approximations (Son and Baral 2001), the idea is to approximate a belief state by a consistent set of fluent literals that are known to be true, and to define transitions considering what definitely holds and what may change at the next state. With such an approximation, the computational complexity of conditional planning becomes NP-complete. Given upper bounds for the height and the number of branches, ASCP generates a conditional plan with one call of an ASP solver based on an intelligent formulation of possible transitions. HCP-ASP does not consider 0-approximations of belief states, but requires that the initial values of fluents that cannot be identified by any sensing action are known in advance, decided nondeterministically (e.g., by a disjunction over the possible values), or specified with respect to some assumptions (e.g., by defaults). Since HCP-ASP is compilation-based, it calls an ASP solver multiple times to build a conditional plan.

There is a variety of recent work on hybrid planners that combine motion planning with classical planners. Some of them are based on modifications/introductions of search algorithms for motion/task planning (Gravot et al. 2005; Hauser and Latombe 2009; Kaelbling and LozanoPérez 2013; Lagriffoul et al. 2014; Gaschler et al. 2013; Srivastava et al. 2014), some of them are based on formal methods (Plaku 2012; Dantam et al. 2016), and some of them are based on modification of representation of domains (Caldiran et al. 2009; Hertle et al. 2012; Erdem et al. 2011; Erdem et al. 2016). HCP-ASP is similar to the latter group, since feasibility checks are embedded in action descriptions via external atoms (in the spirit of semantic attachments in theorem proving (Weyhrauch 1978)) without having to modify the classical planners or motion planners. We refer the reader to the recent studies (Lagriffoul et al. 2014; Erdem et al. 2016) surveying and empirically analyzing some of these approaches. 


\section{Answer Set Programming}

Answer Set Programming (ASP) (Brewka et al. 2016) is a form of knowledge representation and reasoning paradigm oriented towards solving combinatorial search problems as well as knowledge-intensive problems. The idea of ASP is to represent a problem as a "program" whose models (called "answer sets" (Gelfond and Lifschitz 1991)) correspond to the solutions. The answer sets for the given program can be computed by special implemented systems called ASP solvers, such as CLINGO (Gebser et al. 2014).

We consider ASP programs (i.e., nondisjunctive HEX programs (Eiter et al. 2005)) that consist of rules of the form

$$
\text { Head } \leftarrow A_{1}, \ldots, A_{m}, \text { not } A_{m+1}, \ldots, \text { not } A_{n}
$$

where $n \geq m \geq 0$, Head is an atom or $\perp$, and each $A_{i}$ is an atom or an external atom. A rule is called a fact if $m=n=0$ and a constraint if Head is $\perp$. Please see Appendix A for more details.

We model robotic action domains as ASP programs as described in (Erdem et al. 2016a). We use the ASP solver CLINGO with relevant feasibility checkers to compute hybrid plans (i.e., discrete task plans integrated with continuous feasibility checks).

There are several important aspects of ASP that are worth emphasizing here as they are closely relevant to our contributions in this paper. First, it is possible to embed the results of external computations into ASP programs using special constructs, called "external atoms", in the spirit of semantic attachments in theorem proving (Weyhrauch 1978). For instance, the ASP rule

$$
\perp \leftarrow a t\left(r, x_{1}, y_{1}, t\right), \text { goto }\left(r, x_{2}, y_{2}, t\right), \text { not \&path_exists }\left[x_{1}, y_{1}, x_{2}, y_{2}\right]()
$$

expresses that, at any step $t$ of the plan, a robot $r$ cannot move from $\left(x_{1}, y_{1}\right)$ to $\left(x_{2}, y_{2}\right)$ if it is not known that a collision-free trajectory between them exists. Here, collision check is done by the external atom \& path_exists $\left[x_{1}, y_{1}, x_{2}, y_{2}\right]()$ implemented in $\mathrm{C}++$, utilizing the bidirectional RRT algorithm (Kuffner Jr and LaValle 2000). External atoms allow us to compute hybrid plans.

Second, it is possible to express defaults in ASP thanks to "default negation" not. The defaults allow us to model our assumptions about a dynamic robotic world with partial observability. For instance, consider a kitchen where a service robot sets up the table for a meal. Some locations are partially observable, but the table is fully observable (i.e., the robot knows what is on the table). The following ASP rule

$$
\neg a t(o, \text { Table }, t) \leftarrow \text { not at }(o, \text { Table }, t)
$$

expresses that by default the objects $o$ in a kitchen are not assumed to be on the table unless they are known to be on the table.

Third, it is possible to express nondeterministic choice in ASP using "choice expressions" with "cardinality constraints". Choice expressions help us to model occurrences and non-occurrences of actions. For instance, the following ASP rule

$$
\{\operatorname{sense}(a t(o), t)\}
$$

expresses that the action of sensing the location of an object can occur any time. Choice expressions with cardinality constraints help us to model nondeterministic effects of sensing actions. For instance, the following ASP rule

$$
1\{\operatorname{at}(o, l, t+1): \operatorname{loc}(l)\} 1 \leftarrow \operatorname{sense}(\operatorname{at}(o), t)
$$

describes that if sensing is applied to check the location of an object $o$ (i.e., $\operatorname{sense}(\operatorname{atObj}(o), t)$ ), 
then we know that the object $o$ is at one of the possible locations $l$; here, the location $l$ is nondeterministically chosen by the ASP solver.

Fourth, it is possible to express "unknowns" using "cardinality expressions"; e.g., the rule

$$
\neg a t(o, m, t) \leftarrow\{a t(o, l, t): \operatorname{loc}(l)\} 0
$$

expresses that if object's location is not known (i.e., $\{a t(o, l, t): \operatorname{loc}(l)\} 0)$ then it definitely can not be at a robot's hand $m$.

Fifth, we can express "weak constraints" to minimize, e.g., the number of sensing actions:

$$
\sim \operatorname{senseAct}(t)[2 @ 2, t]
$$

Finally, the incremental computation of an answer set by an ASP solver, like CLINGO, allows for minimization of makespans (i.e., lengths) of plans.

\section{Hybrid Conditional Plans}

A hybrid conditional plan can be characterized as a labeled directed tree $(V, E)$. The set $V=$ $V_{a} \cup V_{s}$ of vertices consists of two types of vertices. The vertices in $V_{a}$ (called actuation vertices) characterize hybrid actuation actions (e.g., the robot's navigation and manipulation actions integrated with feasibility checks). The vertices in $V_{s}$ (called sensing vertices) characterize sensing actions or information gathering actions in general (e.g., finding out or checking the location of an object). The leaves of the tree are in $V_{a}$.

The set $E$ of edges between vertices in $V$ characterizes the order of actions: an edge $(x, y)$ expresses that the action denoted by the vertex $x$ is to-be executed before the action denoted by $y$. Each vertex in $V_{a}$ has at most one outgoing edge based on the assumption that the actuation actions are deterministic. Each vertex in $V_{s}$ has at least two outgoing edges. Each sensing action may lead to different outcomes/observations.

Let us denote by $E_{s}$ the set of outgoing edges from vertices in $V_{s}$. Then a labeling function maps every edge $(x, y)$ in $E_{s}$ by a possible outcome of the sensing action characterized by $x$.

\section{A Parallel Algorithm for Computing a Hybrid Conditional Plan}

HCP-ASP computes a hybrid conditional plan utilizing the expressive formalism and efficient solvers of ASP. HCP-ASP first computes a hybrid sequential plan of sensing actions and actuation actions using ASP; this sequential plan characterizes a single branch of the hybrid conditional plan being constructed. After identifying the sensing actions in this plan, for each sensing action and for each outcome of the sensing action, HCP-ASP computes a hybrid conditional plan in parallel. Finally, it combines these hybrid conditional plans as children of the sensing nodes. The overall algorithm is depicted in Algorithm 1. It is important to emphasize the novelties of our algorithm by explaining how it handles some of the challenges.

\subsection{Computation of a single branch of the hybrid conditional plan}

Considering the incomplete knowledge about the initial state, partial observability of the domain, deciding for (non)occurrences of sensing actions, nondeterministic effects of sensing actions, and necessity of feasibility checks for actuation and sensing actions for robotic applications, make it challenging to compute even a single branch of a hybrid conditional plan. We deal with these challenges by describing the robotic domain and the planning problem in ASP. 


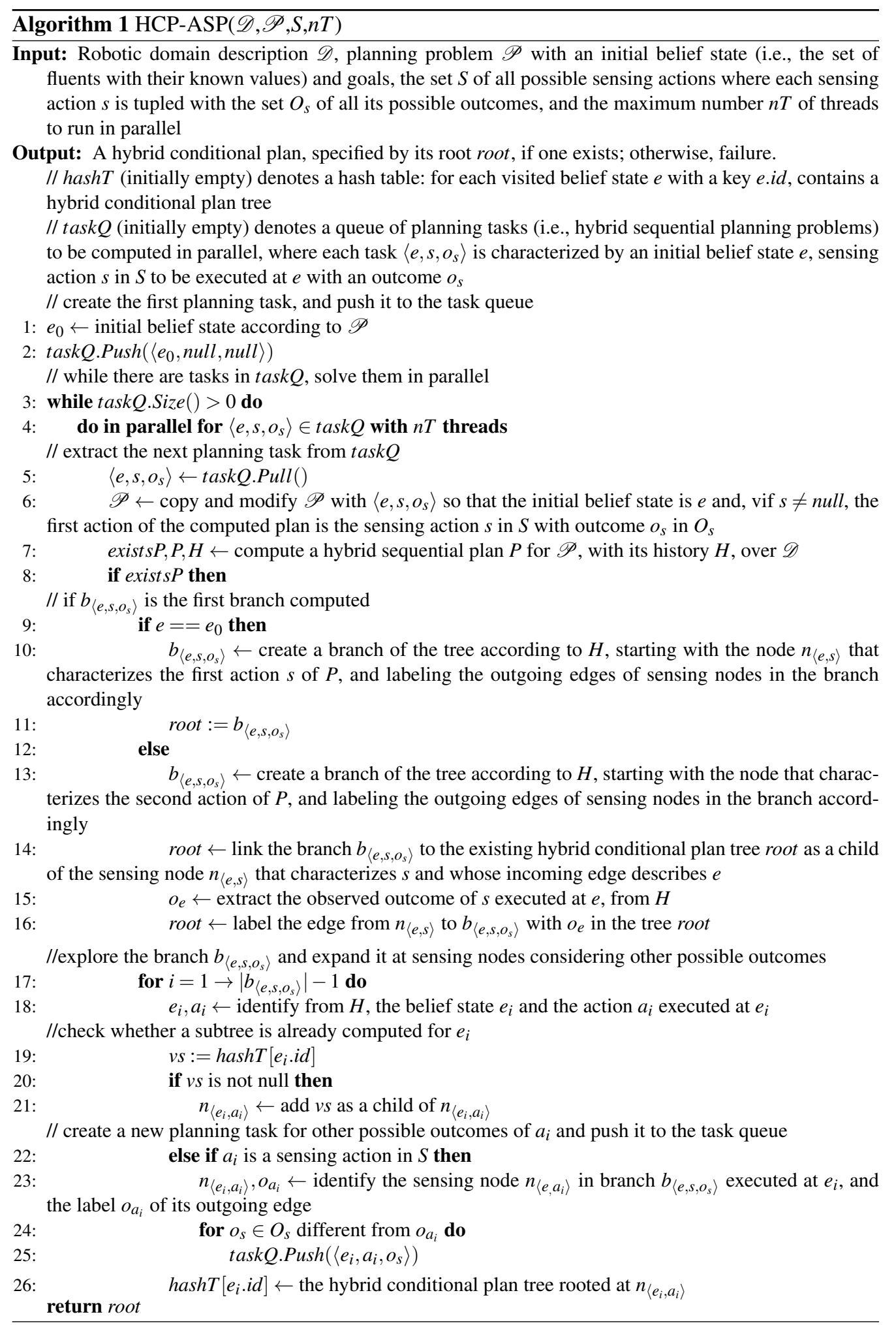


Representing actuation actions. We formalize preconditions and effects of actuation actions in robotic domains as described in (Erdem et al. 2016a). For some examples, please see Appendix B.

Integrating feasibility checks. We integrate low-level feasibility checks into computation of a hybrid sequential plan, utilizing external atoms, as described in Section 3, with respect to different methods of integration, as surveyed in (Erdem et al. 2016).

Representing belief states. We assume that values $v$ of functional fluents $f(\bar{x})$ at step $t$ are represented by atoms of the form $f(\bar{x}, \bar{v}, t)$ in ASP.

We assume that some functional fluents are fully observable (i.e., known by the robot), while some are partially observable (i.e., can be identified by sensing actions). If a functional fluent is fully observable then a uniqueness constraint

$$
\leftarrow 2\left\{f(\bar{x}, \bar{v}, t): \operatorname{dom}_{\bar{x}}(\bar{v}\}\right.
$$

and an existence constraint

$$
\leftarrow\left\{f(\bar{x}, \bar{v}, t): \operatorname{dom}_{\bar{x}}(\bar{v})\right\}
$$

are included in the domain description. If the fluent is partially observable then a uniqueness constraint (3) is added only; the value of the fluent is identified when the relevant sensing action is performed.

For every functional fluent $f(\bar{x})$, if there exist some value $\bar{v}$ such that $f(\bar{x}, \bar{v}, t)$ belongs to an answer set then we believe that $f(\bar{x})$ is known at step $t$. Otherwise, if there is no $\bar{v}$ such that $f(\bar{x})=\bar{v}$ belongs to an answer set for time step $t$, we believe that the value of $f(\bar{x})$ is not known. In that sense, partial assignments of values to fluents represent belief states in our framework.

Representing initial values of fluents. If the initial values of fully observable fluents are known, then they are specified by a set of facts. Otherwise, they are decided nondeterministically (e.g., by a disjunction over the possible values or by a choice rule):

$$
\{f(\bar{x}, \bar{v}, 0)\} \leftarrow \operatorname{dom}_{\bar{x}}(\bar{v})
$$

or specified with respect to some assumptions (e.g., by defaults).

$$
\neg f(\bar{x}, \bar{v}, 0) \leftarrow \text { not } f(\bar{x}, \bar{v}, 0), \operatorname{dom}_{\bar{x}}(\bar{v}) .
$$

If the initial values of partially observable fluents are known, then they are specified by a set of facts.

Representing nondeterministic hybrid sensing actions. We characterize sensing actions by atoms of the form sense $(f(\bar{x}), t)$ which describes an occurrence of a sensing action that determines the value of a unique partially observable fluent $f(\bar{x})$ at time step $t$.

We suppose that sensing actions may occur exogenously at any time, and we represent this assumption by the following choice rule, like in rule (1):

$$
\{\operatorname{sense}(f(\bar{x}), t)\} \text {. }
$$

We suppose that sensing actions sense $(f(\bar{x}), t)$ are not executed if the value of the relevant fluent $f(\bar{x})$ is known. We express this assumption by the following constraint:

$$
\leftarrow \operatorname{sense}(f(\bar{x}), t), 1\left\{f(\bar{x}, \bar{v}, t): \operatorname{dom}_{\bar{x}}(\bar{v})\right\} .
$$

After that, we describe that whenever a sensing action sense $(f(\bar{x}), t)$ is executed, the observed 
outcome $\bar{v}$ is nondeterministically chosen among possible values of $f(\bar{x})$. We express this nondeterministic choice of an outcome using choice expressions and cardinality constraints in ASP, like in rule (2):

$$
1\left\{f(\bar{x}, \bar{v}, t+1): \operatorname{dom}_{\bar{x}}(\bar{v})\right\} 1 \leftarrow \operatorname{sense}(f(\bar{x}), t) .
$$

Sometimes, executability of a sensing action necessitates further conditions to hold. For instance, to check whether a plate is clean or not, the robot has to be holding the plate. The preconditions of a sensing action may involve feasibility checks as well. For instance, checking what is on the table is possible if the camera is "reachable" to a position over the table. This can be expressed by a constraint as follows:

$$
\leftarrow \operatorname{sense}(a t(o), t), \text { not \& reachable }[\text { Table }]() .
$$

Incremental computation of a hybrid sequential plan of sensing actions and actuation actions. Given a domain description and a planning problem as described above, the ASP solver CLINGO can compute a plan to reach a goal state from a possibly incomplete initial state. CLINGO first grounds this whole program and then computes an answer set for the ground program; the answer set characterizes the plan.

We improve this computation (grounding and answer set finding), by ensuring that CLINGO incrementally performs this computation by increasing the upper bound on the maximum time step one by one, but without having to ground the whole theory every time. This is possible thanks to the "incremental mode" of CLINGO.

\subsection{Parallel computation of subtrees}

Our algorithm maintains a queue task $Q$ of tasks $\left\langle e, s, o_{s}\right\rangle$ where $e$ is an initial belief state $e, s$ is a sensing action, $o_{s}$ is an outcome of $s$. Initially, $\operatorname{task} Q$ contains the task $\left\langle e_{0}\right.$, null, null $\rangle$ only (Algorithm 1, Line 2). Each task $\left\langle e, s, o_{s}\right\rangle$ in task $Q$ describes a hybrid sequential planning problem with the initial state $e$ and the goal as same as in the hybrid conditional planning problem $\mathscr{P}$ (Lines 5\&6). This planning problem has further constraints, if $s$ and $o_{s}$ are not null: $s$ is a sensing action executed at belief state $e$ and the outcome of this execution is $o_{s}$ (Line 7). Note that these constraints can be easily expressed in ASP.

For every task $\left\langle e, s, o_{s}\right\rangle$, an ASP solver computes a hybrid sequential plan $P=\left\langle a_{0}, a_{1}, \ldots, a_{k}\right\rangle$ and its history $H=\left\langle b_{0}, a_{0}, b_{1}, a_{1}, \ldots, b_{k}, a_{k}, b_{k+1}\right\rangle$, where $s=a_{0}, e=b_{0}$, each $a_{i}$ is an actuation action or a sensing action executed at a belief state $b_{i}$ and reaching a belief state $b_{i+1}(0 \leq i<k)$, the last action $a_{k}$ is an actuation action, and the last belief state $b_{k+1}$ is a goal state according to $\mathscr{P}$. Such a plan $P$ describes a branch $b_{\left\langle e, s, o_{s}\right\rangle}=\left\langle n_{\left\langle b_{0}, a_{0}\right\rangle}, n_{\left\langle b_{1}, a_{1}\right\rangle}, \ldots, n_{\left\langle b_{k}, a_{k}\right\rangle}\right\rangle$ of the tree that starts at the node $n_{\langle e, s\rangle}=n_{\left\langle b_{0}, a_{0}\right\rangle}$ and ends at the leaf $n_{\left\langle a_{k}, b_{k}\right\rangle}$. For every sensing action $a_{i}$ in $P$, the edge from $n_{\left\langle b_{i}, a_{i}\right\rangle}$ to $n_{\left\langle b_{i+1}, a_{i+1}\right\rangle}$ is labeled by its outcome $o_{a_{i}}$ observed in $b_{i+1}$. Once we compute all the branches that start at a sensing node, we can combine them at that node (Lines 9-16).

The tasks are added to the queue $\operatorname{task} Q$ at different times: for every other outcome of every sensing node in a branch $b_{\left\langle e, s, o_{s}\right\rangle}$, a task is constructed (Lines 17-26). Since the tasks in task $Q$ are not dependent on each other, they are solved in parallel utilizing at most $n T$ threads (Line 4).

Discussion about correctness. Every branch of the tree from the root to a leaf constructed incrementally as described above characterizes a hybrid sequential plan, under the assumptions that a plan can be constructed by merging two plans: $\mathscr{D}$ respects the Markov principle (so actions do not have delayed effects), and $\mathscr{D}$ does not contain any temporal constraints that ensure/prevent 
occurrences of actions or observations of fluents in some order, or any constraints to guarantee some conditions over the whole hybrid sequential plan (e.g., a constraint on the total cost of actions). Indeed, let $P=\left\langle a_{0}, a_{1}, \ldots, a_{k}\right\rangle$ be a hybrid sequential plan computed for a planning problem $\mathscr{P}$ with respect to a domain description $\mathscr{D}$, where each $a_{i}$ is an actuation action or a sensing action $(0 \leq i<k)$ and the last action $a_{k}$ is an actuation action. Let $H=\left\langle b_{0}, a_{0}, b_{1}, a_{1}, \ldots, b_{k}, a_{k}, b_{k+1}\right\rangle$ be a history of this plan, where every action $a_{i}$ is executed at a belief state $b_{i}$ and reaching a belief state $b_{i+1}(0 \leq i<k)$, and the last belief state $b_{k+1}$ is a goal state according to $\mathscr{P}$. For every sensing action $a_{j}$ in $P(0<j<k)$ with an outcome $o_{a_{j}}$ observed at $b_{j+1}$, a task $\left\langle b_{j}, a_{j}, o_{a_{j}}\right\rangle$ is constructed and pushed into task $Q$. Consider one of these tasks: $\left\langle b_{j}, a_{j}, o_{a_{j}}\right\rangle$. Let $P^{\prime}=\left\langle a_{j}=a_{0}^{\prime}, a_{1}^{\prime}, \ldots, a_{k^{\prime}}^{\prime}\right\rangle$ be a hybrid sequential plan, with its history $H^{\prime}=\left\langle b_{j}=b_{0}^{\prime}, a_{0}^{\prime}, b_{1}^{\prime}, a_{1}^{\prime}, \ldots, b_{k^{\prime}}^{\prime}, a_{k^{\prime}}^{\prime}, b_{k^{\prime}+1}^{\prime}\right\rangle$, computed for the planning problem characterized by this task. Then, due to the assumptions mentioned above, the sequence $\left\langle a_{0}, a_{1}, \ldots, a_{j}, a_{1}^{\prime}, \ldots, a_{k^{\prime}}^{\prime}\right\rangle$ of actions is also a hybrid sequential plan computed for a planning problem $\mathscr{P}$, with a history $\left\langle b_{0}, a_{0}, b_{1}, a_{1}, \ldots, b_{j}, a_{j}, b_{1}^{\prime}, a_{1}^{\prime}, \ldots, b_{k^{\prime}}^{\prime}, a_{k^{\prime}}^{\prime}, b_{k^{\prime}+1}^{\prime}\right\rangle:$ the first part of the plan, $\left\langle a_{0}, a_{1}, \ldots, a_{j}\right\rangle$, does not prevent the last part of the plan, $\left\langle a_{1}^{\prime}, \ldots, a_{k^{\prime}}^{\prime}\right\rangle$, and vice versa. Note that these assumptions can be waived if we modify our algorithm slightly by computing every branch of the tree from the root, instead of constructing branches by combining sub-branches: every task $\left\langle b_{j}, a_{j}, o_{a_{j}}\right\rangle$ is understood as a planning problem obtained from $\mathscr{P}$ by adding constraints to ensure that $\left\langle b_{0}, a_{0}, b_{1}, a_{1}, \ldots, b_{j}, a_{j}\right\rangle$ is a prefix of the history of the plan and that $o_{a_{j}}$ is observed right after execution of $a_{j}$. On the other hand, note that the hybrid sequential plans computed from the root have larger makespans (and thus they lead to computations over larger ASP programs) compared to the plans computed from intermediate states.

\subsection{Avoiding recomputation of subtrees}

Our algorithm keeps a hash table hashT that maps a belief state to a hybrid conditional plan computed from that state (Lines 18-21,26). In this way, identical parts of a hybrid conditional plan are not computed repeatedly. This then allows the representation of a hybrid conditional plan more compactly as a directed acyclic graph (DAG).

For some domains, when the desired goal relies on a subset of fluents only, we define "equivalence classes" of belief states according to these fluents. This allows us to utilize the hash table with respect to these equivalence classes. If belief states $e$ and $e^{\prime}$ belong to the same equivalence class, and if a hybrid conditional plan is computed for $e$, then we utilize this tree also for $e^{\prime}$ despite their differences.

For instance, consider the colorball planning domain (Albore et al. 2009) used as a benchmark for conditional planners. In this domain, the goal is to dispose a given set of balls with unknown locations and colors into thrash boxes according to their colors. As long as a ball is disposed into the trash, its color or location does not affect the rest of the plan anymore. So we can safely say that two different belief states are equivalent if they only differ in color and/or location of any ball that is trashed. Fortunately, we can specify this information in ASP (in a separate file) by identifying what parts of belief states could be ignored as follows:

$$
\begin{aligned}
& \text { redundant }(\text { color }(b, c, t)) \leftarrow \operatorname{trashed}(b, t) \\
& \text { redundant }(\text { location }(b, l, t)) \leftarrow \operatorname{trashed}(b, t) .
\end{aligned}
$$

When a ball $b$ is thrashed, its color $c$ and location $l$ are irrelevant to belief state comparisons. Here redundant is a reserved predicate for our planner to identify partial belief states for comparisons. 


\subsection{Advantages of hybridity of conditional plans}

Since each branch of a conditional plan depicts a possible execution of actuation/sensing actions to reach a goal, it is essential that these actions are checked against relevant feasibility constraints in real-world applications. In robotics applications, these constraints are required for collision-free navigation and reachable/graspable manipulation, as depicted in Figure 2 with two conditional plans computed for a robotics scenario, where two bimanual mobile manipulators are responsible for setting up a kitchen table: none of the branches of the non-hybrid plan (Figure 2(a)) is executable in real world, since the actuation actions (denoted red) are not feasible; whereas every branch of the hybrid plan (Figure 2(b)) is feasible in real world. See Appendix C, for more details about the domain.



(a)



(b)

Fig. 2: Two hybrid conditional plans generated (a) without and (b) with feasibility checks.

\section{Experimental Evaluations}

Comparisons with the hybrid compilation-based conditional planner HCPlan We have compared HCP-ASP (with CLINGO 4.5.4) with HCPlan (with CCALC using MINISAT 2.2.0) over the robotics kitchen table setting domain of (Nouman et al. 2016). This domain models a dynamic service robotics scenario, where a bimanual mobile manipulator is responsible for setting up a kitchen table by navigating around a kitchen to manipulate kitchenware. The robot can perform sensing actions to identify the locations of objects, cleanliness of objects, and to determine the type of food requested by the user. The goal is to for the robot to setup the table for lunch, with proper kitchenware for the requested food type. This domain includes several low-level feasibility checks, such as kinematic reachability, bimanual motion planning for robot arms, and collision-free navigation planning. For our experiments, we have constructed 12 instances of this domain, with solutions whose heights range over 16-33 and branching factor ranges over 2-4.

All experiments are conducted on a PC workstation running Ubuntu 16.04 on $162.4 \mathrm{GHz}$ Intel E5-2665 CPU cores with 64GB memory and an SSD hard drive. As suggested by the PRE integration method of (Erdem et al. 2016), the feasibility checks are performed in advance as in (Nouman et al. 2016) and cached in a hash table, for both planners. A 5 hour threshold is used to compute plans, and computations that exceed this threshold are marked as Time Out (TO). For 
parallel implementations of HCP-ASP and HCPlan, the maximum number of available threads are limited to 20. HCP-ASP generates a more compact representation of the tree as a DAG due to re-use of subtrees whereas HCplan generates a tree. Therefore, we report the tree sizes of plans.

Table 2 summarizes some results of our experiments. For each instance and the computed hybrid conditional plan, we present the maximum branch length (i.e., makespan of the longest hybrid sequential plan), the number of sensing nodes in the tree (i.e., total number of sensing actions in the plan), the number of leaves of the tree (i.e., number of different hybrid sequential plans represented in the tree), the number of nodes in the tree (i.e., total number of actuation and sensing actions in the plan), the computation time in seconds (i.e., walltimes for the threads with longest computations). The instances are arranged in ascending order of tree sizes.

HCplan uses CCALC with incremental grounding. Also, HCP-ASP uses CLINGO with incremental grounding. Therefore, both planners try to minimize the makespans of hybrid sequential plans. Although the planners start with a shortest hybrid sequential plan of the same makespan, due to the occurrences of different actions in the plans in different orders, the trees may have different heights in the end. This can be observed from the maximum branch lengths.

Using weak constraints, HCP-ASP further tries to minimize the number of sensing actions, once it minimizes actuation actions. Due to this, the trees computed by HCP-ASP have less number of sensing actions, and often with less number of leaves and nodes. Depending how the tree changes, the computation time may increase due to such further optimizations.

Due to parallel computations of hybrid sequential plans and subtrees, the computation times reflect in general the computation time of the longest hybrid sequential plan. Indeed, for the longest branches of the trees of Instance 12, CLINGO takes much longer time than CCALC.

Comparisons with non-hybrid compilation-based conditional planners We have also compared HCP-ASP with non-hybrid compilation-based planners CLG, DNFct and PO-PRP, over two standard benchmarks (Albore et al. 2007; Albore et al. 2009). In the colorball domain, the goal is to dispose a given set of $x$ balls with unknown locations and colors into thrash boxes according to their colors; the environment is represented as a $n \times n$ grid. The location and color of a ball can be observed when the agent and the ball are in the same cell. In the doors domain, the goal is to reach a goal location by moving in a $n \times n$ grid with hidden doors; open doors can be detected by sensing actions. We have formalized these domains in ASP, by allowing multi-valued sensing

Table 2: Comparison of HCP-ASP with HCPlan on the table setting domain.

\begin{tabular}{|c||r|r|r|r|r|r|r|r|r|r|}
\hline \multirow{2}{*}{ Scen. } & \multicolumn{2}{|c|}{ Max Branch Length } & \multicolumn{2}{c|}{ No. Sensing Nodes } & \multicolumn{2}{|c|}{ No. of Leaves } & \multicolumn{2}{|c|}{ Tree Size } & \multicolumn{2}{|c|}{ Time } \\
\hline & HCplan & HCP-ASP & HCplan & HCP-ASP & HCplan & HCP-ASP & HCplan & HCP-ASP & HCplan & HCP-ASP \\
\hline 1 & 17 & 17 & 4 & 3 & 12 & 11 & 147 & 114 & 19.66 & 18.85 \\
\hline 2 & 21 & 21 & 11 & 9 & 19 & 17 & 168 & 186 & 25.91 & 47.70 \\
\hline 3 & 21 & 20 & 15 & 8 & 35 & 25 & 306 & 243 & 31.38 & 26.79 \\
\hline 4 & 23 & 23 & 20 & 24 & 31 & 32 & 294 & 283 & 35.24 & 106.77 \\
\hline 5 & 23 & 22 & 31 & 24 & 45 & 32 & 398 & 329 & 41.66 & 180.80 \\
\hline 6 & 21 & 25 & 31 & 26 & 72 & 40 & 566 & 377 & 33.8 & 53.82 \\
\hline 7 & 26 & 26 & 49 & 54 & 102 & 68 & 771 & 615 & 45.73 & 164.48 \\
\hline 8 & 26 & 26 & 53 & 54 & 91 & 68 & 676 & 615 & 38.96 & 164.11 \\
\hline 9 & 29 & 24 & 103 & 58 & 222 & 96 & 1535 & 698 & 53.88 & 239.26 \\
\hline 10 & 29 & 31 & 231 & 224 & 347 & 280 & 2296 & 1844 & 67.99 & 202.25 \\
\hline 11 & 29 & 29 & 227 & 156 & 349 & 218 & 2524 & 1591 & 76.28 & 280.52 \\
\hline 12 & 33 & 31 & 1483 & 576 & 2178 & 773 & 16119 & 5164 & 390.29 & 973.28 \\
\hline
\end{tabular}


outcomes and concurrency of actuation actions. Since CLG generates a tree, while DNFct and PO-PRP generate DAGs, both DAG sizes and tree sizes are reported for HCP-ASP.

Table 3 presents the results of these experiments. CB $x-n$ (resp. Doors $n$ ) denotes instances of the colorball (resp. doors) domain. For each instance, the size of DAG and/or tree (the number of nodes), the maximum branch length, and total computation time in seconds are reported in the table. MO indicates the planner did not have enough memory to complete the problem. Note that the available version of PO-PRP has a 5GB memory limit.

The results indicate that, for most of the scenarios, HCP-ASP generates shorter trees and smaller DAGs. HCP-ASP scales in time better then CLG and PO-PRP, with a performance very close to that of DNFct. While PO-PRP shows remarkable time performance in available instances, the time and memory scaling of this planner seem poorer than others. HCP-ASP outperforms other planners in generated DAG size which has high correlation with time and memory efficiency. The DAGs computed by HCP-ASP are much smaller than the trees they represent. This shows the usefulness of identifying equivalence classes to detect similar states.

Comparisons with ASCP As discussed in Section 2, although not compilation-based, the offline non-hybrid conditional planner ASCP also uses ASP to compute conditional plans. Therefore, we have compared these two ASP-based conditional planners, over one of the benchmarks of ASCP: Bomb in the Toilet with Sensing Actions (BTS) (Weld et al. 1998). In our experiments with ASCP, we have directly used the ASP encoding of BTS transformed from $\mathscr{A}_{K}^{c}$ with the ASP solver CLINGO. According to the results (presented in Appendix D), finding a tree with one call of CLINGO (using ASCP) takes more time, compared to computing and combining the branches of the tree in parallel (using HCP-ASP). For instance, for $m=17$, it takes more than an hour to compute a tree with ASCP whereas it takes about a second for HCP-ASP.

Evaluations of different versions of HCP-ASP Using the colorball and doors domains, we also experimentally evaluate the following three versions of HCP-ASP: $v_{0}$ (base planner with parallelism, revisited states, and incremental planning), $v_{1}$ ( $v_{0}$ improved with equivalence classes), $v_{2}$ ( $v_{1}$ improved with more efficient representation of the domain to capture multi-valued sensing outcomes and concurrency of actuation actions). The version $v_{2}$ is used in Tables 2 and 3 .

Table 3: Comparison of offline compilation-based conditional planners.

\begin{tabular}{|c|c|c|c|c|c|c|c|c|c|c|c|c|c|}
\hline & \multicolumn{3}{|c|}{ CLG } & \multicolumn{3}{|c|}{ DNFct } & \multicolumn{3}{|c|}{ PO-PRP } & \multicolumn{4}{|c|}{ HCP-ASP } \\
\hline & Tree & Max & Time & DAG & Max & Time & DAG & Max & Time & Tree & \begin{tabular}{|l|} 
DAG \\
\end{tabular} & Max & Time \\
\hline & Size & Depth & {$[\mathrm{sec}]$} & Size & Depth & [sec] & Size & Depth & {$[\mathrm{sec}]$} & Size & Size & Depth & {$[\mathrm{sec}]$} \\
\hline B 3-1 & 95 & 21 & 0.04 & 35 & 19 & 0.03 & 89 & 23 & 0.02 & 81 & 51 & 17 & 0.7 \\
\hline B 3-2 & 2641 & 34 & 0.63 & 609 & 40 & 0.47 & 1655 & 48 & 0.1 & 1800 & 415 & 28 & 2 \\
\hline В 3-3 & 60924 & 48 & 17.86 & 8021 & 57 & 8.45 & 3247 & 73 & 3.9 & 38977 & 3395 & 41 & 29.8 \\
\hline B 3-4 & 29235 & 61 & 542 & 93379 & 72 & 268 & $\mathrm{MO}$ & $\mathrm{MO}$ & MO & 805768 & 32593 & 48 & 928 \\
\hline CB 4-1 & 295 & 52 & 0.2 & 67 & 36 & 0.04 & 271 & 46 & 0.06 & 242 & 100 & 33 & 1.4 \\
\hline CB 4-2 & 20050 & 74 & 11.75 & 1928 & 70 & 2.85 & 12432 & 90 & 1.46 & 14067 & 1362 & 58 & 9.9 \\
\hline B $4-3$ & 136920 & 100 & 966 & 39678 & 100 & 227 & $\mathrm{MO}$ & $\mathrm{MO}$ & $\mathrm{MO}$ & 708580 & 27942 & 84 & 681 \\
\hline B 5-1 & 586 & 65 & 0.59 & 117 & 68 & 0.95 & 556 & 78 & 0.1 & 524 & 172 & 63 & 4 \\
\hline B 5-2 & 72817 & 107 & 100 & 5153 & 113 & 18 & 49767 & 169 & 15.22 & 53576 & 3479 & 98 & 38.5 \\
\hline CB 5-3 & TO & TO & TO & 134008 & 157 & 2338 & $\mathrm{MO}$ & MO & MO & 4718549 & 13879 & 149 & 13173 \\
\hline B 9-1 & 3385 & 197 & 44.45 & 351 & 184 & 47.28 & 2607 & 279 & 1.1 & 3210 & 621 & 211 & 77.5 \\
\hline CB 9-2 & 00499 & 312 & 4918 & 43554 & 360 & 1564 & MO & MO & MO & 1238013 & 42213 & 378 & 6779 \\
\hline Doors 5 & 144 & 24 & 0.18 & 146 & 26 & 0.05 & 105 & 28 & $\overline{0.06}$ & 156 & 68 & 27 & 1.2 \\
\hline Doors 7 & 2153 & 51 & 3.38 & 2193 & 53 & 0.79 & 1282 & 60 & 0.34 & 2381 & 179 & 59 & 6.5 \\
\hline Doors 9 & 46024 & 95 & 188 & 44998 & 89 & 29.76 & 23897 & 104 & 7.92 & 52065 & 381 & 109 & 47.4 \\
\hline Doors 11 & $\mid 1213759$ & 135 & 19228 & 1161651 & 124 & 1239 & $\mathrm{MO}$ & $\mathrm{MO}$ & MO & 1242179 & 776 & 167 & 265 \\
\hline
\end{tabular}


Table 4 presents the experimental results. For each instance, the size of DAG, the maximum branch length, parallel efficiency (i.e., ratio of sequential computation time to $(20 \times$ parallel computation time)), and the total computation time in seconds are reported in the table.

The colorball domain well demonstrates the effects of improvements in different versions of HCP-ASP, while the doors domain is useful to evaluate the potential of partial state comparisons, where a problem can be divided into sub-problems. Since the doors domain is very simple, it does not allow for $v_{2}$, as there is no sensing outcome that can converted to multi-valued format and there is only a single actuation action not allowing for concurrency.

Comparison of computation times of $v_{0}$ and efficiency values indicate that parallelism reaches its full potential with up to $99.9 \%$ efficiency as the problem size gets larger. This is expected as the number of tasks that can be computed at any time depends on the number of sensing actions and the outcomes at each branch. From the results of $v_{1}$, one can observe that utilization of equivalence classes result in highly reduced DAG size and number of branches explored, significantly improving the computation time.

From the results of $v_{2}$, one can observe that the use of multi-valued functional fluents and thus multi-valued sensing outcomes (e.g., identifying the color of a ball) decrease the length of branches compared to the case with boolean fluents and thus sensing outcomes (e.g., identifying that a ball has a specified color), since outcome of a single multi-valued sensing action can be obtained by an exponential number of boolean sensing actions. Similarly, the use of concurrent actions reduce the length of plans, since multiple actions can be executed at the same time.

\section{Conclusion}

We have introduced an offline parallel compilation-based hybrid conditional planner, called HCP-ASP, utilizing various features and utilities of ASP solver CLINGO. From the representation point of view, compared to many existing offline compilation-based conditional planners, HCP-ASP allows multi-valued functional fluents, multi-valued sensing actions, explicit representation of occurrences, preconditions and nondeterministic effects of sensing actions, weak

Table 4: Comparison of different versions of HCP-ASP

\begin{tabular}{|l||r|r|r|r||r|r|r|r||r|r|r|r|}
\hline & \multicolumn{4}{|c||}{$v_{0}$} & \multicolumn{4}{|c||}{$v_{1}$} & \multicolumn{3}{|c|}{$v_{2}$} \\
\hline & DAG & Max & Eff. & Time & DAG & Max & Eff. & Time & DAG & Max & Eff. & Time \\
& Size & Depth & $\%$ & sec & Size & Depth & $\%$ & sec & Size & Depth & $\%$ & sec \\
\hline CB 3-1 & 63 & 20 & 10 & 0.8 & 63 & 20 & 10 & 1.1 & 51 & 17 & 11 & 0.7 \\
CB 3-2 & 728 & 39 & 60 & 3.3 & 635 & 39 & 58 & 3.6 & 415 & 28 & 78 & 2 \\
CB 3-3 & 6960 & 52 & 40 & 93 & 4780 & 52 & 34 & 90 & 3395 & 41 & 97 & 29.8 \\
CB 3-4 & 113466 & 67 & 89 & 6738 & 55904 & 69 & 26 & 5835 & 32593 & 48 & 100 & 928 \\
\hline CB 4-1 & 117 & 37 & 11 & 1.6 & 121 & 40 & 12 & 1.8 & 100 & 33 & 12 & 1.4 \\
CB 4-2 & 1934 & 66 & 64 & 12.7 & 1503 & 69 & 57 & 12.8 & 1362 & 58 & 93 & 9.9 \\
CB 4-3 & 34074 & 101 & 98 & 788 & 24500 & 102 & 84 & 635 & 27942 & 84 & 99.8 & 681 \\
\hline CB 5-1 & 181 & 68 & 8 & 4.6 & 181 & 68 & 8 & 5.5 & 172 & 63 & 10 & 4 \\
CB 5-2 & 4939 & 121 & 65 & 65.5 & 3872 & 118 & 49 & 76.6 & 3479 & 98 & 96 & 38.5 \\
CB 5-3 & 123623 & 166 & 99 & 10023 & 109157 & 162 & 98 & 8168 & 138793 & 149 & 100 & 13173 \\
\hline CB 9-1 & 634 & 220 & 8 & 102 & 634 & 220 & 8 & 106 & 621 & 211 & 10 & 77.5 \\
CB 9-2 & 45584 & 397 & 96 & 8916 & 43235 & 397 & 96 & 9504 & 42213 & 378 & 98 & 6779 \\
\hline Doors 5 & 158 & 27 & 13 & 1 & 68 & 27 & 10 & 1.2 & & & \\
Doors 7 & 2410 & 65 & 54 & 7.6 & 179 & 59 & 13 & 6.5 & & & \\
Doors 9 & 49381 & 109 & 99 & 523 & 381 & 109 & 19 & 47.4 & & & \\
Doors 11 & TO & TO & TO & TO & 776 & 167 & 30 & 265 & & & \\
\hline
\end{tabular}


constraints to minimize sensing actions or actuation actions, concurrency of actuation actions, and integration of feasibility checks into action descriptions. From the computation point of view, HCP-ASP re-uses earlier computations due to partial state comparisons via equivalence classes specified in ASP, generates trees in a more compact form of DAGs, performs incremental computations of branches (i.e., hybrid sequential plans), and utilizes parallel computation of branches in a subtree as well as parallel computation of subtrees. Due to these novelties, HCPASP not only provides a new conditional planner with a more expressive representation language and promising computational performance, but also provides a useful tool for hybrid robotic applications with incomplete knowledge and partial observability. Our planner HCP-ASP and the benchmarks are available at http://cogrobo. sabanciuniv. edu/?p=1073.

Acknowledgments. This work is partially supported by the Science Academy (BAGEP). We thank T. C. Son and H. Geffner for useful discussions about HCP-ASP, and A. Albore, T. C. Son and C. Muise for their helps with CLG, DNFct, PO-PRP respectively.

\section{Appendix A ASP Programs}

We consider ASP programs (i.e., nondisjunctive HEX programs (Eiter et al. 2005)) that consist of rules of the form

$$
\text { Head } \leftarrow A_{1}, \ldots, A_{m}, \text { not } A_{m+1}, \ldots, \text { not } A_{n}
$$

where $n \geq m \geq 0$, Head is an atom or $\perp$, and each $A_{i}$ is an atom or an external atom. HEX programs can be extended by allowing classical negation $\neg$ in front of atoms. A rule is called a fact if $m=n=0$ and a constraint if Head is $\perp$.

An external atom is an expression of the form $\& g\left[y_{1}, \ldots, y_{k}\right]\left(x_{1}, \ldots, x_{l}\right)$ where $y_{1}, \ldots, y_{k}$ and $x_{1}, \ldots, x_{l}$ are two lists of terms (called input and output lists, respectively), and $\& g$ is an external predicate name. Intuitively, an external atom provides a way for deciding the truth value of an output tuple depending on the extension of a set of input predicates. External atoms allow us to embed results of external computations into ASP programs. They are usually implemented in a programming language of the user's choice, like $\mathrm{C}++$. For instance, the following rule

$$
\begin{aligned}
\perp \leftarrow \text { at }\left(r, x_{1}, y_{1}, t\right), \text { goto }\left(r, x_{2}, y_{2}, t\right), & \text { not \&path_exists }\left[x_{1}, y_{1}, x_{2}, y_{2}\right]()
\end{aligned}
$$

is used to express that, at any step $t$ of the plan, a robot $r$ cannot move from $\left(x_{1}, y_{1}\right)$ to $\left(x_{2}, y_{2}\right)$ if there is no collision-free trajectory between them. Here collision check is done by the external predicate \&path_exists implemented in C++, utilizing the bidirectional RRT (Rapidly Exploring Random Trees) (Kuffner Jr and LaValle 2000) as in the OMPL (Sucan et al. 2012) library.

In addition to the classical negation, ASP considers another sort of negation: "negation as failure" (denoted not, and often called "default negation"). Intuitively, $\neg p$ expresses that we know that $p$ does not hold, whereas not $p$ expresses that we do not know that $p$ holds. This second sort of negation empowers ASP to express our assumptions (called "defaults") when we do not have complete knowledge. For instance, we can express that "every object $o$ in a kitchen is assumed to be on the counter unless they are known to be on the table" by the following ASP rule

$$
\text { at }(o, \text { Counter }, t) \leftarrow \text { not at }(o, \text { Table }, t) .
$$

In ASP, we use special constructs to express nondeterministic choices, cardinality constraints, 
and optimization statements. For instance, the following ASP rule (called a "choice rule")

$$
\{\operatorname{sense}(\operatorname{at}(o), t)\}
$$

contains the choice expression sense $(a t(o), t)$ in the head. For every object $o$ and time step $t$, this choice expression describes a subset of the set $\{\operatorname{sense}(a t(o), t)\}$. Therefore, this rule expresses that, for every object $o$ and time step $t$, the action of sensing that the location of $o$ may occur at $t$.

The following ASP constraint (called a "cardinality constraint")

$$
\leftarrow 2\{\operatorname{atRob}(l, t): \operatorname{robloc}(l)\}
$$

contains the cardinality expression $2\{a t R o b(l, t): \operatorname{robloc}(l)\}$ in the body. For every time step $t$, this expression describes subsets of the set $\{\operatorname{atRob}(l, t): \operatorname{robloc}(l)\}$ whose cardinality is at least 2 . Therefore, this constraint is used to ensure that, for every time step $t$, the robot cannot be at two different locations at $t$.

The following ASP expression (called an "optimization statement")

$$
\# \text { minimize }[\operatorname{cost}(r, c, t): \operatorname{robot}(r)=c]
$$

is used to minimize the sum of all costs $c$ of robotic actions performed in a plan, where costs of actions performed by robot $r$ at time step $t$ are defined by atoms of the form $\operatorname{cost}(r, c, t)$.

A version of external atoms (where predicate names are not passed as input arguments), and all the constructs described above are supported by the ASP solver CLINGO used as part of HCP-ASP. For more information about the input language of CLINGO, we refer the reader to CLINGO's manual: https://sourceforge. net/projects/potassco/files/guide/2. 0/guide-2.0.pdf (June 18, 2017).

\section{Appendix B Hybrid Classical Planning in ASP}

Classical planning considers complete knowledge (under full observability) over a dynamic domain. A dynamic domain is defined by means of fluent constants and (actuation) action constants: A world state can be characterized by an interpretation of fluent constants, whereas an action is characterized by an interpretation of action constants. Then, dynamic domains under full observability can be modeled as transition systems - directed graphs where nodes denote the world states of the domain, and edges denote the transitions between these states caused by occurrences or nonoccurrences of actions in that domain. Note that such transition systems respect the Markov principle (i.e., actions do not have delayed effects).

Given an initial state $s_{0}$, goal conditions $G$, and a nonnegative integer $k$, classical planning asks for a sequence $P=\left\langle a_{0}, a_{1}, \ldots, a_{k-1}\right\rangle$ of actions, which characterizes a path $X=\left\langle s_{0}, s_{1}, \ldots, s_{k-1}, s_{k}\right\rangle$ from $s_{0}$ to a goal state $s_{k}$ in this transition system such that every edge $\left(s_{i}, s_{i+1}\right)$ in the path characterizes an occurrence of action $a_{i}$. This sequence $P$ of actions is called a plan, with makespan $k$. The history $H=\left\langle s_{0}, a_{0}, s_{1}, a_{1}, \ldots, s_{k-1}, a_{k-1}, s_{k}\right\rangle$ of a plan describes the path $X$ by depicting also the relevant actions. Classical planning is NP-complete for polynomially bounded plans (Erol et al. 1995).

For robotic domains, to ensure executability of classical plans, e.g., to ensure continuous movements along collision-free trajectories, further checks need to be performed. This requires combining classical planning with feasibility checks. We call this problem hybrid classical planning. As discussed in Section 2 of the main paper, there are different methods of integrating 
planning with feasibility checks. We consider solving hybrid classical planning problems in ASP, using HEX programs, with respect to appropriate methods of integration (Erdem et al. 2016).

Representing hybrid action domains in ASP We formalize hybrid dynamic domains in ASP, under full observability and as a transition system, in the spirit of (Erdem et al. 2016a). Such a description of a hybrid dynamic domain in ASP relies on three important forms of rules.

For a formula $H$ and an index $i$ (for time step), let us denote by $H(i)$ the formula obtained from $H$ by replacing every atom $q$ by $q(i)$. Intuitively, $H(i)$ expresses that the formula $H$ holds at time step $i$.

Effect rules: Direct outcomes of actions are expressed with effect rules of the form

$$
E(i+1) \leftarrow A(i), F(i)
$$

where $A$ is a conjunction of action atoms, $E$ is a fluent literal, and $F$ is conjunction of fluent literals. This rule indicates that if the actions in $A$ are executed at time step $i$ where $F$ holds then at the next state $E$ holds. For instance, the following effect rule describes an effect of a "move" action of a mobile robot $r$ navigating to a location $l$ at time step $i$ :

$$
a t(r, l, i+1) \leftarrow \operatorname{move}(r, l, i) .
$$

It expresses that, as a direct effect of this action, the location of robot $r$ changes to $l$ at the next time step $i+1$.

Precondition rules: Preconditions of actions are expressed with precondition rules of the form

$$
\leftarrow A(i), F(i), \text { not } G(i)
$$

where $A$ is a conjunction of action atoms, and $F$ and $G$ are conjunctions of fluent literals. The precondition rule above expresses that, to execute an action $A$ at time step $i$ at a state where $F$ holds, the action's preconditions $G$ must hold. For instance, according to the following precondition rule

$$
\leftarrow \operatorname{move}(r, l, i), \text { at }(r, l, i)
$$

action move $(r, l)$ is possible if the robot is not already at the destination location $l$.

Hybrid rules: A hybrid rule is a rule where the right hand side of $\leftarrow$ includes external atoms. External atoms (Eiter et al. 2005) are not fluent or action constants; their truth values are computed externally (out of ASP).

These rules are important for robotics applications since low-level feasibility checks for each action can be computed externally and then integrated into transition system description by means of external atoms. For instance, the following hybrid precondition rule ensures that, at time step $i$, a robot $r$ can move from its current location $x$ to its destination location $l$ if there is a collision-free trajectory between them:

$$
\leftarrow \text { at }(r, x, i), \text { move }(r, l, i), \text { not \&move_is_feasible }[r, l, x]() .
$$

The external atom \&move_is_feasible $[r, l, x]()$ passes $r, l, x$ as inputs to the external computation (e.g., a Python program) that calls a motion planner to check the existence of a collision free trajectory for $r$ from $x$ to $l$, and then returns the result of the computation to the precondition rule. 
Defining planning problems in ASP Once the domain is described in ASP, we can specify the initial state of the world in different ways, e.g., by a set of facts, like

$$
\text { atRob(Table, } 0) \text {, }
$$

by choice rules accompanied with constraints, like

$$
\begin{aligned}
& \{\operatorname{atRob}(l, 0)\} \\
& \leftarrow \operatorname{not} \text { atRob }(\text { Table }, 0),
\end{aligned}
$$

or by assumptions, like

$$
\text { atRob }(\text { Table }, 0) \leftarrow \neg \text { atRob }(\text { Table }, 0) .
$$

We can describe the goal conditions by a set of rules, and add constraints to ensure their reachability, as in the examples below:

$$
\begin{aligned}
& \text { goal } \leftarrow \text { atRob }(\text { Table }, t) \\
& \leftarrow \text { not goal. }
\end{aligned}
$$

Then, with a domain description and planning problem description, plans of actuation actions can be computed using the ASP solver CLINGO.

\section{Appendix C An Example for Hybrid Conditional Planning: Kitchen Domain}

As a benchmark for hybrid conditional planning, we consider a dynamic service robotics scenario, where a bimanual mobile manipulator is responsible for setting up a kitchen table, as depicted in Figure C 1. This domain is introduced in (Nouman et al. 2016): "The mobile manipulator can navigate around the kitchen to pick up and place objects as long as collision free trajectories exist. Kitchenware, such as mugs, spoons, knives, plates may be found in cabinets or may be left on other flat surfaces, such as counter tops or shelves. In the kitchen, there also exists a faucet to clean kitchenware as required. Finally, there is a kitchen table, where the proper kitchenware must be placed on to comply with table setting etiquette."

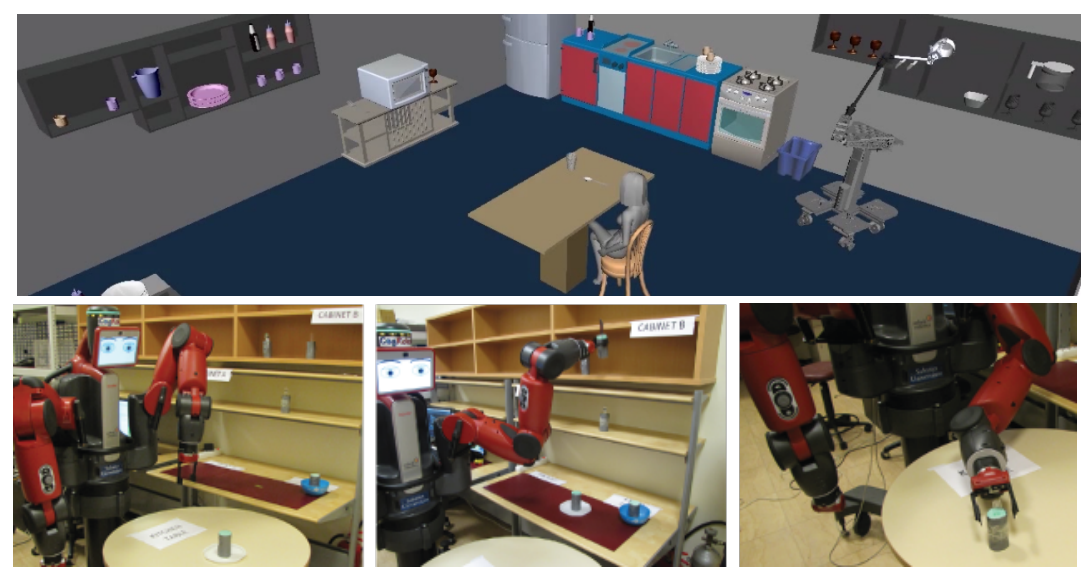

Fig. C 1: The robot is manipulating a bowl from Cabinet B in dynamic simulation (top) and a fork during physical implementation (bottom).

In this domain, there are four actuation actions: goto, pickup, placeon and clean. For the feasibility of these actions, existence of a collision-free trajectory is implemented based on 
OMPL (Sucan et al. 2012) (to be used as a precondition of goto action), while reachability, graspability and inverse kinematics checks are implemented based on OPENRAVE (Diankov 2010) (to be used as preconditions of pickup and placeon actions).

This domain contains three types of uncertainties. First, the person might have different food preferences (e.g., soup, pizza, salad), which can only be revealed when directly communicated with the user during plan execution. Second, the locations of some kitchenware may not be known by the robot during the planning phase. These locations can be reliably gathered only if the robot actively searches for these objects when it needs to use them. Third, the cleanliness/dirtiness of the objects may not be known in advance for sure. Along these lines, three sensing actions are considered: checkFoodType, checkLoc and checkisClean.

An ASP description of this domain presented to HCP-ASP, in the input language of CLINGO, is provided in Figures $\mathrm{C} 2-\mathrm{C} 8$. The description consists of three parts, appropriate for incremental grounding (and thus incremental computation of plans), and preceded by the expressions \#program base, \#program step(t), and \#program check(t). Intuitively, the first part describes the domain predicates and the general knowledge about the world at time step 0; so it is instantiated once. The second part describes the states at time step $t$ and transitions of the world for time steps $t-1$ and $t$. Here, the value of $t$ increases one by one starting from 1 until a plan is found; so this part is instantiated incrementally. This incremental grounding guarantees finding a plan with a minimum length. The direct/indirect effects of actions, inertia, and action occurrences are defined in the second part. The third part describes all the constraints to be checked at every time step $t$. Here, the value of $t$ also increases one by one starting from 0 until a plan is found; so this part is instantiated incrementally as well. The preconditions of actions, state constraints, transition constraints, concurrency constraints, and the goal are defined in the third part.

The actuation actions are defined as described in Appendix B, whereas the sensing actions are defined as described in Section 5 of the main paper.

\section{Appendix D Experimental comparison of HCP-ASP with ASCP}

As discussed in Section 2 of the main paper, although not compilation-based, the offline nonhybrid conditional planner ASCP also uses ASP to compute conditional plans. Therefore, we have compared these two ASP-based conditional planners, over one of the benchmarks of ASCP: Bomb in the Toilet with Sensing Actions (BTS) (Weld et al. 1998). In this domain, it has been alarmed that there is a bomb in the toilet. There are $m$ suspicious packages, and one of them contains the bomb. The bomb can be defused by dunking the package with bomb into the toilet; dunking a package clogs the toilet and flushing the toilet unclogs it. The existence of a bomb in a package can be sensed by a metal detector, by a dog to sniff the bomb, or by an x-ray machine. Initially, the bomb is armed and the toilet is not clogged; the goal is that the bomb is disarmed and the toilet is not clogged.

We have experimented with ASCP using the ASP encoding of BTS (bt_3sa.smo) ${ }^{1}$ transformed from $\mathscr{A}_{K}^{c}$ with the ASP solver CLINGO. The results of experiments for $m=10,11, . ., 17$ are shown in Table D 1; the computation time for ASCP does not contain the time for transformation.

${ }^{1}$ https://www.cs.nmsu.edu/ tson/ASPlan/Sensing/test/bt_3sa.smo. 
According to these results, finding a tree with one call of CLINGO (using ASCP) takes more time, compared to computing and combining the branches of the tree in parallel (using HCPASP). For instance, for $m=17$, it takes more than an hour to compute a tree with ASCP whereas it takes about a second for HCP-ASP.

Table D 1: Comparison of ASCP with HCP-ASP.

\begin{tabular}{|c|c|r|r|r|}
\hline No of Package & Max Depth & Tree Size & $\begin{array}{r}\text { ASCP Time } \\
\text { [sec] }\end{array}$ & (parallel with 20 threads) [sec] \\
\hline 10 & 10 & 19 & 21 & 0.4 \\
11 & 11 & 21 & 21 & 0.5 \\
12 & 12 & 23 & 46 & 0.6 \\
13 & 13 & 25 & 433 & 0.5 \\
14 & 14 & 27 & 406 & 0.7 \\
15 & 15 & 29 & 1953 & 0.9 \\
16 & 16 & 31 & 2896 & 0.8 \\
17 & 17 & 33 & 5807 & 1.0 \\
\hline
\end{tabular}

\section{References}

Albore, A., Palacios, H., And Geffner, H. 2007. Fast and informed action selection for planning with sensing. In Proc. of CAEPIA. 1-10.

Albore, A., Palacios, H., AND GefFner, H. 2009. A translation-based approach to contingent planning. In Proc. of IJCAI. 1623-1628.

Baral, C., Kreinovich, V., AND Trejo, R. 1999. Computational complexity of planning and approximate planning in presence of incompleteness. In Proc. of IJCAI. 948-955.

BONET, B. AND GEFFNER, H. 2011. Planning under partial observability by classical replanning: Theory and experiments. In Proc. of IJCAI. 1936-1941.

BRAFMAN, R. I. AND SHANI, G. 2012. Replanning in domains with partial information and sensing actions. JAIR 45, 565-600.

Brewka, G., Eiter, T., AND TRUszCZynski, M. 2016. Answer set programming: An introduction to the special issue. AI Magazine 37, 3, 5-6.

Bryce, D., KAmbhampati, S., AND Smith, D. E. 2006. Planning graph heuristics for belief space search. JAIR 26, 35-99.

Caldiran, O., Haspalamutgil, K., OK, A., Palaz, C., Erdem, E., and Patoglu, V. 2009. Bridging the gap between high-level reasoning and low-level control. In Proc. of LPNMR.

Dantam, N. T., Kingston, Z. K., Chaudhuri, S., AND KAVRaki, L. E. 2016. Incremental task and motion planning: A constraint-based approach. In Proc. of RSS.

Diankov, R. 2010. Automated construction of robotic manipulation programs. Ph.D. thesis, Carnegie Mellon University, Robotics Institute.

Eiter, T., IANNI, G., SCHINDlAUer, R., AND TOMPITS, H. 2005. A uniform integration of higher-order reasoning and external evaluations in answer-set programming. In Proc. of IJCAI. 90-96.

Erdem, E., Gelfond, M., ANd LeOne, N. 2016a. Applications of answer set programming. AI Magazine 37, 3, 53-68.

Erdem, E., Haspalamutgil, K., Palaz, C., Patoglu, V., and Uras, T. 2011. Combining highlevel causal reasoning with low-level geometric reasoning and motion planning for robotic manipulation. In Proc. of ICRA.

Erdem, E., PAtoglu, V., ANd SchÜller, P. 2016. A systematic analysis of levels of integration between high-level task planning and low-level feasibility checks. AI Commun. 29, 2, 319-349.

Erol, K., NAU, D. S., AND Subrahmanian, V. S. 1995. Complexity, decidability and undecidability results for domain-independent planning. Artif. Intell. 76, 1-22, 75-88.

Gaschler, A., Petrick, R. P., Giuliani, M., Rickert, M., And Knoll, A. 2013. KVP: A knowledge of volumes approach to robot task planning. In Proc. of IROS. 
Gebser, M., Kaminski, R., Kaufmann, B., And Schaub, T. 2014. Clingo = ASP + control: Preliminary report. In Technical Communications of ICLP. Vol. 14(4-5). TPLP, Online Supplement.

GELFOND, M. AND LifSCHITZ, V. 1991. Classical negation in logic programs and disjunctive databases. New Generation Computing 9, 365-385.

Giunchiglia, E., Lee, J., Lifschitz, V., MCCAin, N., AND Turner, H. 2004. Nonmonotonic causal theories. Artif. Intell. 153, 49-104.

Goldman, R. P. AND Boddy, M. S. 1996. Expressive planning and explicit knowledge. In Proc. of AIPS, 110-117.

Gravot, F., CAmbon, S., And Alami, R. 2005. aSyMov:A Planner that deals with intricate symbolic and geometric problems. In Proc. of ISRR, 100-110.

HaUser, K. AND LATOMBe, J.-C. 2009. Integrating task and PRM motion planning: Dealing with many infeasible motion planning queries. In BTAMP at ICAPS.

Hertle, A., Dornhege, C., Keller, T., And Nebel, B. 2012. Planning with semantic attachments: An object-oriented view. In Proc. of ECAI. 402-407.

HOFFMANN, J. AND BRAFMAN, R. I. 2005. Contingent planning via heuristic forward search witn implicit belief states. In Proc. of ICAPS. 71-80.

KAelbling, L. P. AND LOZANo-PÉREZ, T. 2013. Integrated task and motion planning in belief space. IJRR 32, 9-10, 1194-1227.

Komarnitsky, R. AND Shani, G. 2016. Computing contingent plans using online replanning. In Proc. of AAAI. 3159-3165.

KUFFNER JR, J. AND LAVALLE, S. 2000. RRT-connect: An efficient approach to single-query path planning. In Proc. of ICRA. 995-1001.

Lagriffoul, F., Dimitrov, D., Bidot, J., SAfFiotti, A., And Karlsson, L. 2014. Efficiently combining task and motion planning using geometric constraints. IJRR 33, 14, 1726-1747.

Maliah, S., BRAfMAN, R. I., KARPAS, E., AND Shani, G. 2014. Partially observable online contingent planning using landmark heuristics. In Proc. of ICAPS.

Muise, C. J., Belle, V., AND MCIlraith, S. A. 2014. Computing contingent plans via fully observable non-deterministic planning. In Proc. of AAAI. 2322-2329.

Nouman, A., Yalciner, I. F., ERdem, E., ANd PATOGLU, V. 2016. Experimental evaluation of hybrid conditional planning for service robotics. In Proc. of ISER.

Peot, M. A. And Smith, D. E. 1992. Conditional nonlinear planning. In Proc. of AIPS. 189-197.

Petrick, R. P. A. And BACChus, F. 2002. A knowledge-based approach to planning with incomplete information and sensing. In Proc. of AIPS. 212-222.

PLAKU, E. 2012. Planning in discrete and continuous spaces: From LTL tasks to robot motions. In Proc. of TAROS. 331-342.

Pryor, L. AND Collins, G. 1996. Planning for contingencies: A decision-based approach. JAIR 4, 287-339.

Son, T. C. AND BARAL, C. 2001. Formalizing sensing actions: A transition function based approach. Artif. Intell. 125(1-2), 19-91.

Srivastava, S., Fang, E., Riano, L., Chitnis, R., Russell, S., And Abbeel, P. 2014. Combined task and motion planning through an extensible planner-independent interface layer. In Proc. of ICRA.

Sucan, I. A., Moll, M., And KavraKi, L. E. 2012. The open motion planning library. Robotics \& Automation Magazine, IEEE 19, 4, 72-82.

To, S. T., Son, T. C., AND Pontelli, E. 2011. Contingent planning as AND/OR forward search with disjunctive representation. In Proc. of ICAPS.

TU, P. H., SON, T. C., AND BARAL, C. 2007. Reasoning and planning with sensing actions, incomplete information, and static causal laws using answer set programming. TPLP 7(4), 377-450.

Warren, D. H. D. 1976. Generating conditional plans and programs. In Proc. of AISB. 344-354.

Weld, D. S., Anderson, C. R., and Smith, D. E. 1998. Extending Graphplan to Handle Uncertainty \& Sensing Actions. In Proc. of AAAI. 897-904.

Weyhrauch, R. W. 1978. Prolegomena to a theory of formal reasoning. Tech. rep., Stanford University. 


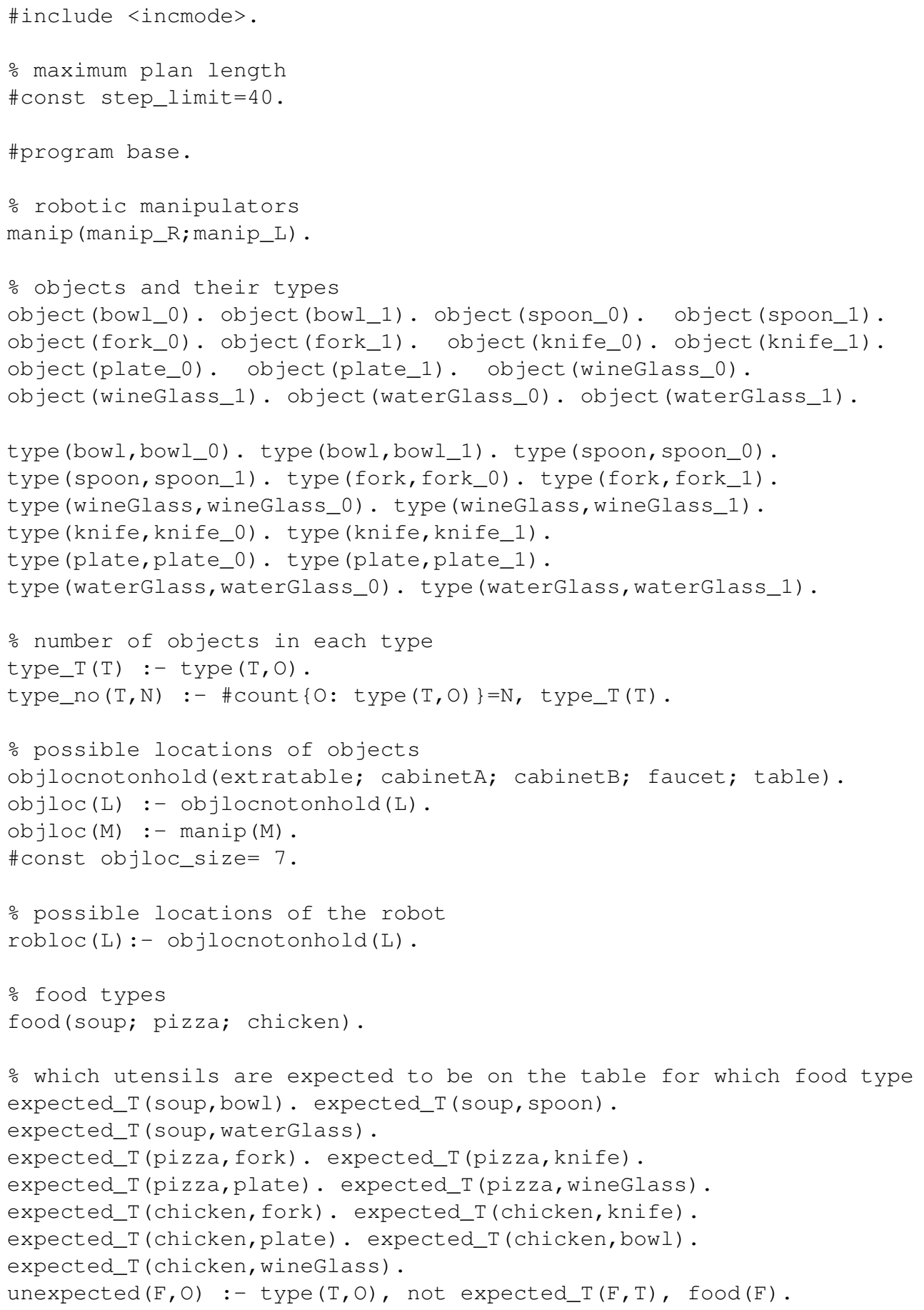

Fig. C 2: Kitchen table setting domain presented to HCP-ASP, in the input language of CLINGO: Part 1 Domain predicates. 


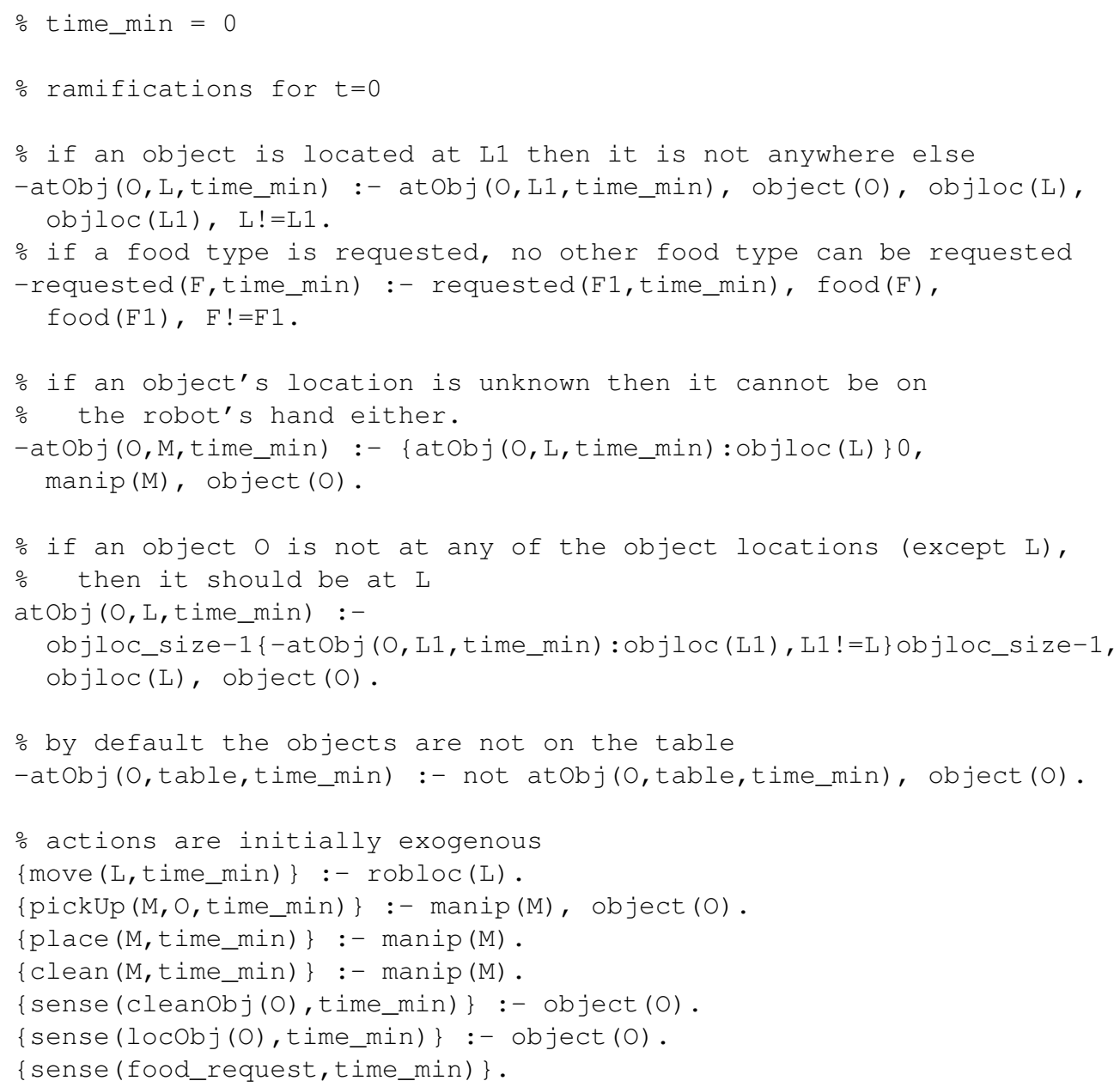

Fig. C 3: Kitchen table setting domain presented to HCP-ASP, in the input language of CLINGO: Part 2 State constraints and possible action occurrences initially. 


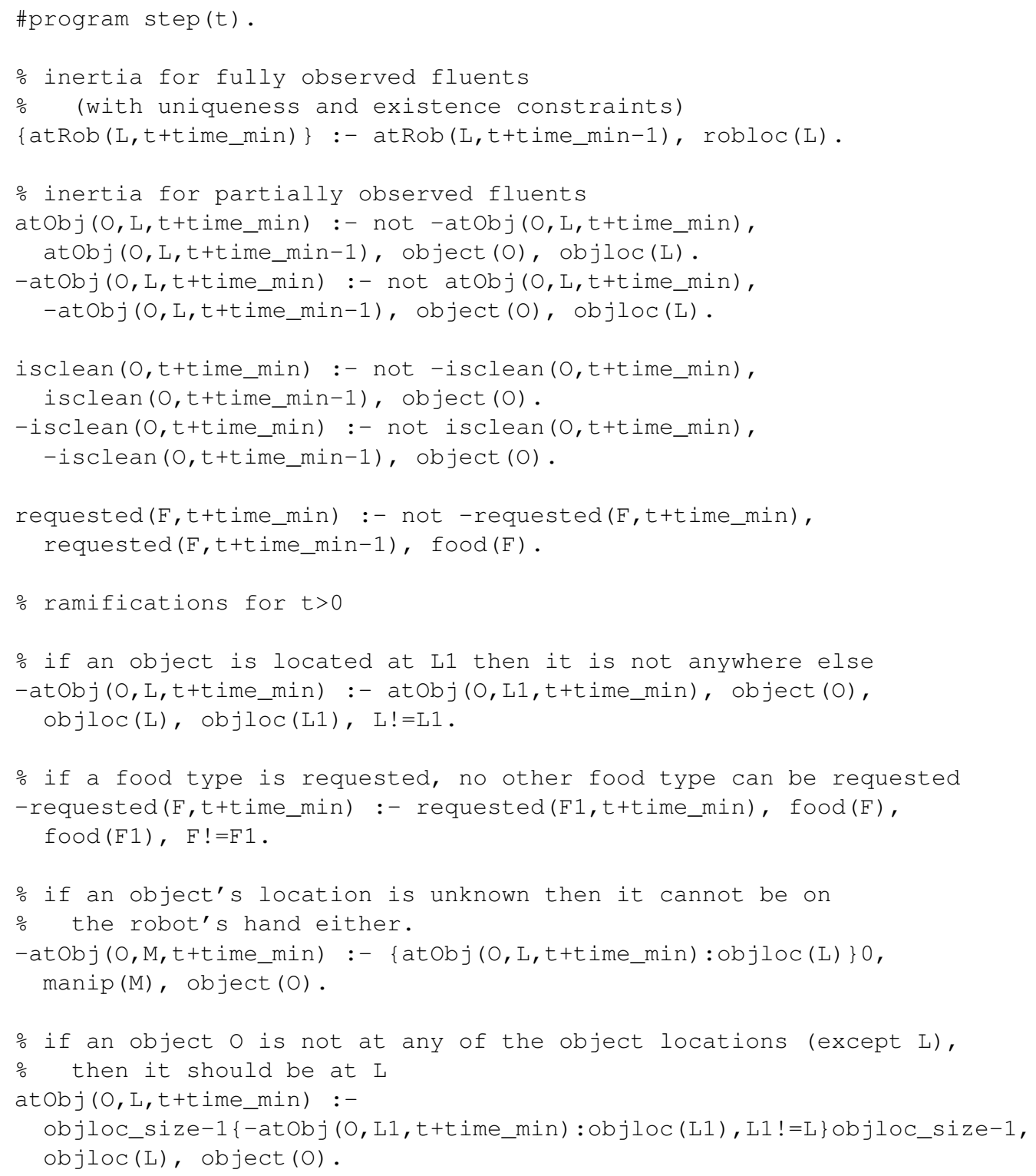

Fig. C 4: Kitchen table setting domain presented to HCP-ASP, in the input language of CLINGO: Part 3 Inertia and ramifications. 


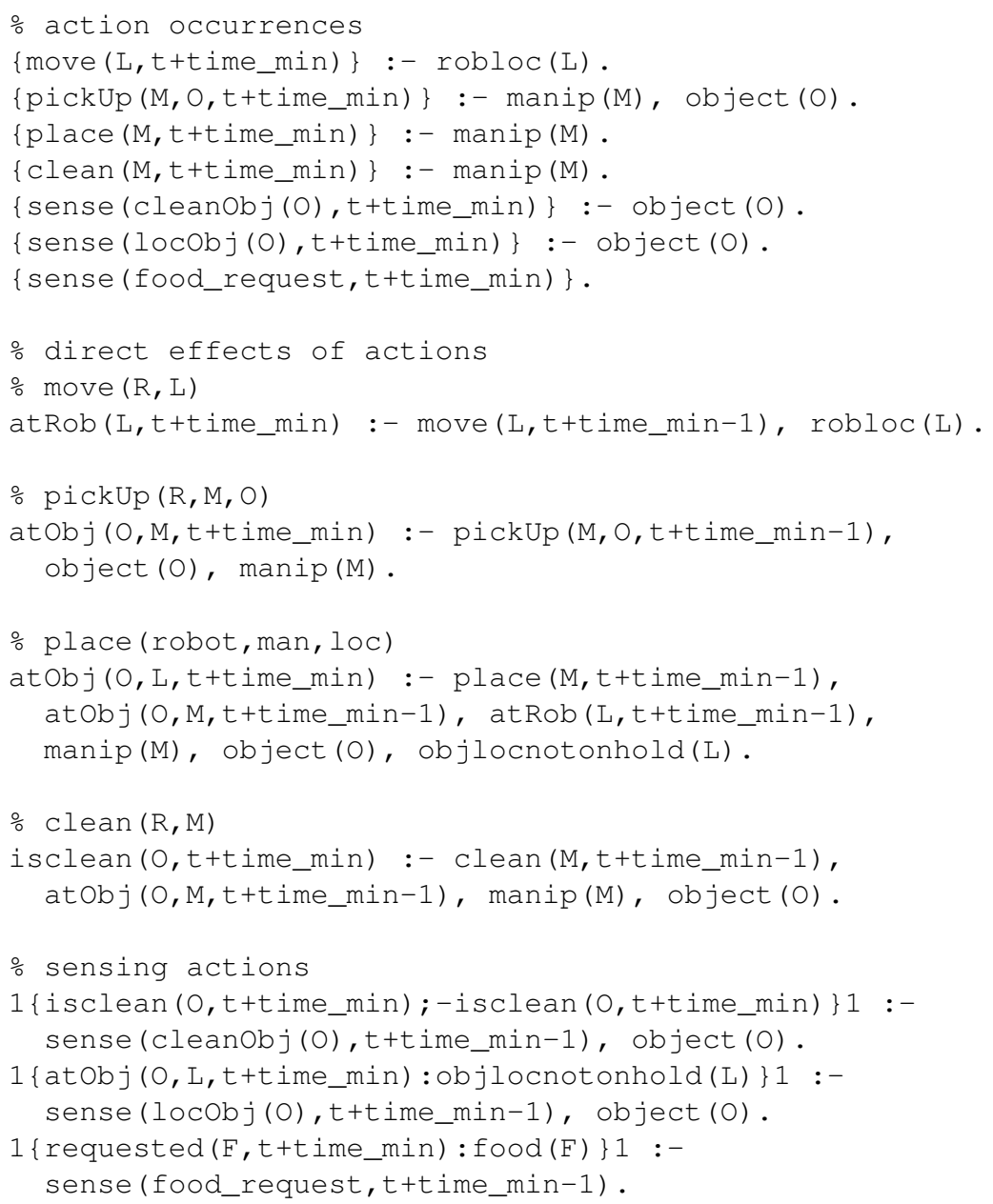

Fig. C 5: Kitchen table setting domain presented to HCP-ASP, in the input language of CLINGO: Part 4 Action occurrences and their direct effects. 




Fig. C 6: Kitchen table setting domain presented to HCP-ASP, in the input language of CLINGO: Part 5 State constraints and preconditions of actions. 


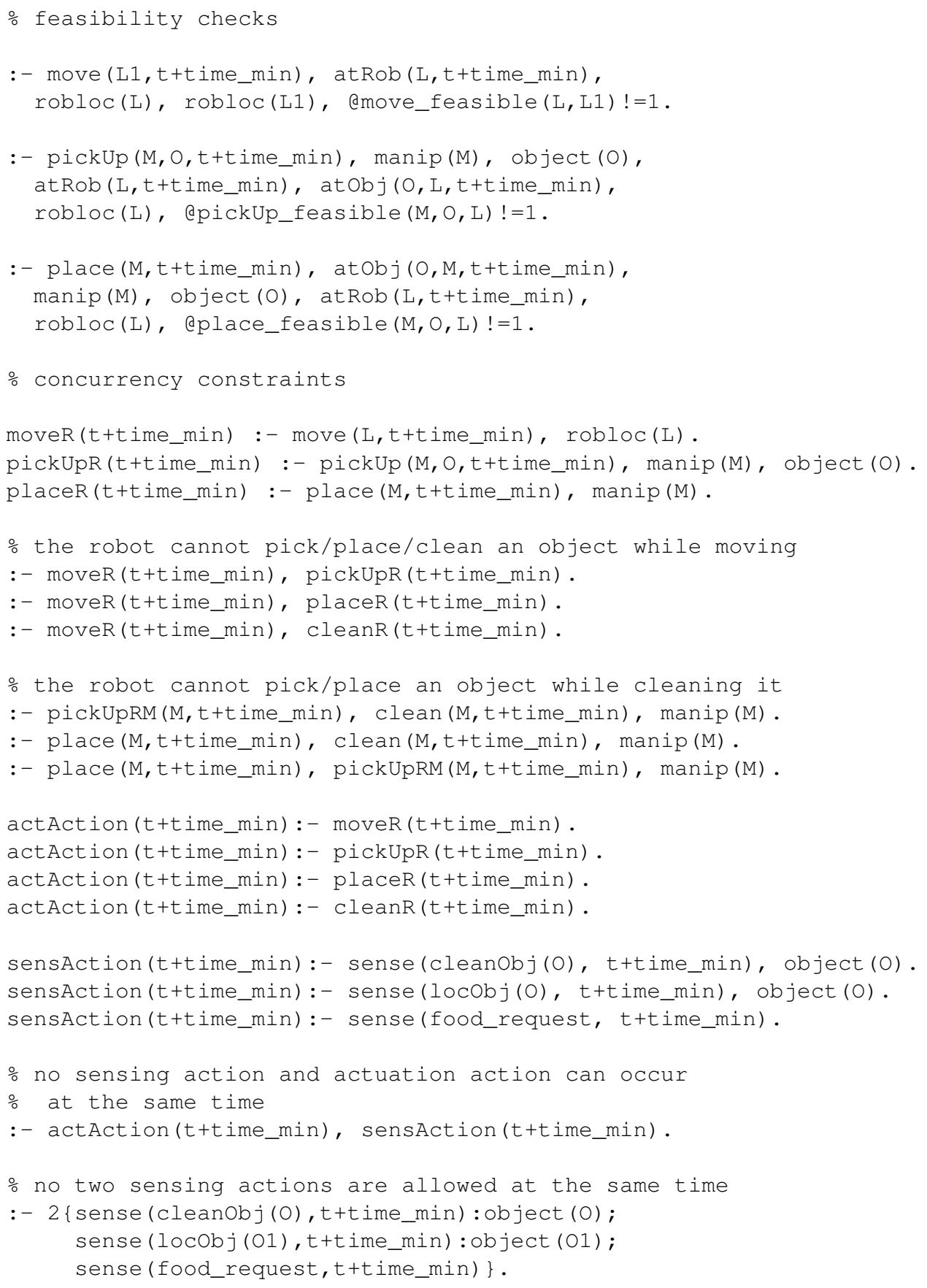

Fig. C 7: Kitchen table setting domain presented to HCP-ASP, in the input language of CLINGO: Part 6 Feasibility checks and concurrency constraints. 


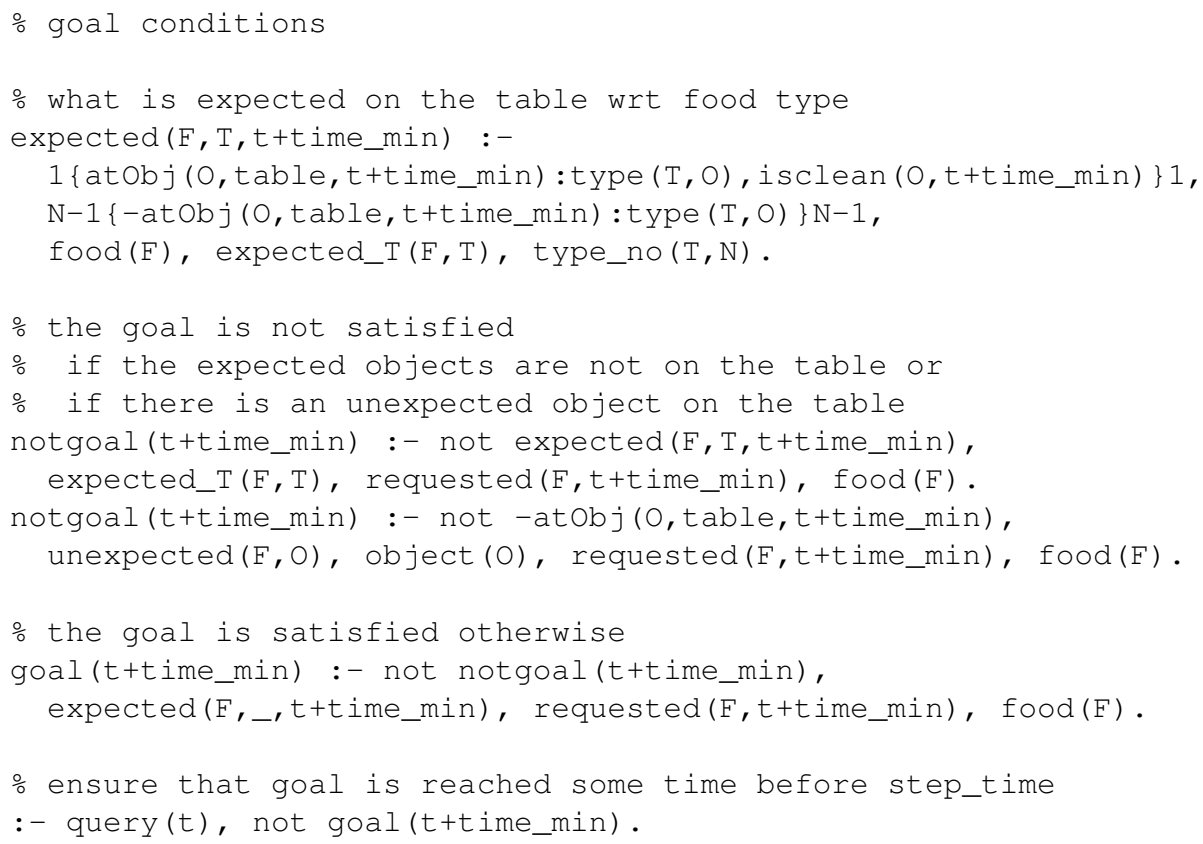

Fig. C 8: Kitchen table setting domain presented to HCP-ASP, in the input language of CLINGO: Part 7 Goal conditions. 